\title{
Cryoablation and cementoplasty of a pathologic fracture in the sternum
}

\author{
John A Swan ${ }^{1}$, MD, FRCPC, David M Liu ${ }^{1}$, MD, FRCPC, Paul W Clarkson ${ }^{2}$, MBChB, Frcsc, Peter L Munk ${ }^{1}$, MDCM, FRCPC
}

\begin{abstract}
A 49-year-old man with metastatic melanoma and pathologic fracture of the sternum was deemed to be a poor candidate for general anaesthesia. He suffered severe pain and range of motion limitation that did not respond to narcotic therapy. Ultimately, the lesion was managed with computed tomography-guided cryoablation and subsequent cementoplasty, and saw good initial clinical results.
\end{abstract}

Keywords: cementoplasty, cryoablation, melanoma, pathologic fracture, sternum

\section{INTRODUCTION}

Bone metastases are common in patients with disseminated malignancy, causing considerable morbidity. As with any other part of the skeleton, the sternum can be affected. Traditionally, radiotherapy has been the treatment for metastases at this site, but in recent times, cement injection combined with radiofrequency ablation (RFA) has been utilised with success. ${ }^{(1-3)}$ Herein, we report our experience using cryoablation in combination with cementoplasty for the treatment of a pathologic fracture of the sternum, which resulted from melanoma.

\section{CASE REPORT}

A 49-year-old man with proven metastatic melanoma presented with central chest pain that was exacerbated on inspiration and motion of the upper extremities. Pre-procedural lateral chest radiograph revealed a displaced sternal fracture (Fig. 1). Severe postural pain over the sternum was experienced by the patient when he sat upright. Computed tomography (CT) imaging was performed, and it demonstrated a lytic lesion measuring $18 \mathrm{~mm} \times 22 \mathrm{~mm}$ in the sternum, approximately $2 \mathrm{~cm}$ below the sternal angle, through which there was a pathologic fracture with approximately $5 \mathrm{~mm}$ anterior displacement of the inferior fracture fragment (Fig. 2). The patient had developed narcotic tolerance and his symptoms did not respond to increased doses of narcotics.

An interventional radiologist was consulted and a palliative procedure was requested. Cryoablation of the tumour at the fracture site was performed, followed by cementoplasty for stabilisation of the fracture. As the patient was being treated for pneumonia and had compromised respiratory function, the department of anaesthesia determined that the patient was unsuitable for general anaesthetic. The procedure was therefore performed under conscious sedation. Cryoablation was chosen over RFA, as the former is a less painful procedure.

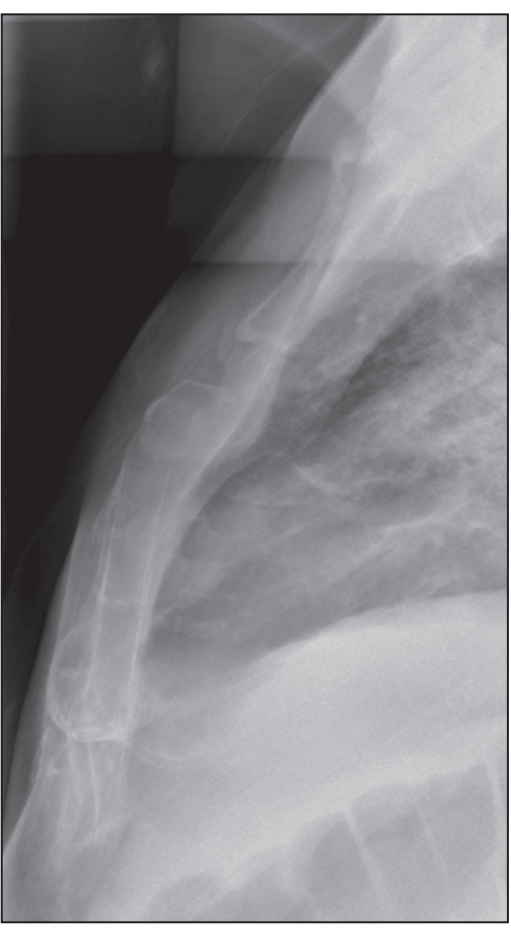

Fig. 1 Pre-procedural lateral chest radiograph (coned-in view of the sternum) shows a displaced sternal fracture.

On obtaining informed consent, the patient was prepared and draped in sterile fashion. An anaesthesiologist was present to provide conscious sedation and monitor the patient's vitals during the procedure. Local anaesthesia was provided with $1 \%$ lidocaine. Under CT guidance, a $13 \mathrm{~F}$ Cook $^{\circledR}$ vertebroplasty needle $\left(\right.$ Cook $^{\circledR}$ Medical Inc, Bloomington, IN, USA) was inserted through a small dermatotomy in the left chest wall, into the lesion. The needle was used as a coaxial guide for the Galil IceSphere ${ }^{\circledR}$ cryoprobe (Galil Medical Inc, Arden Hills, MN, USA), which was advanced fully into the lesion (Fig. 3). The coaxial needle was then withdrawn to expose the cryoprobe. Latex gloves filled with warm water were placed on the skin

${ }^{1}$ Department of Radiology, ${ }^{2}$ Department of Orthopaedics, University of British Columbia and Vancouver General Hospital, Vancouver, Canada 

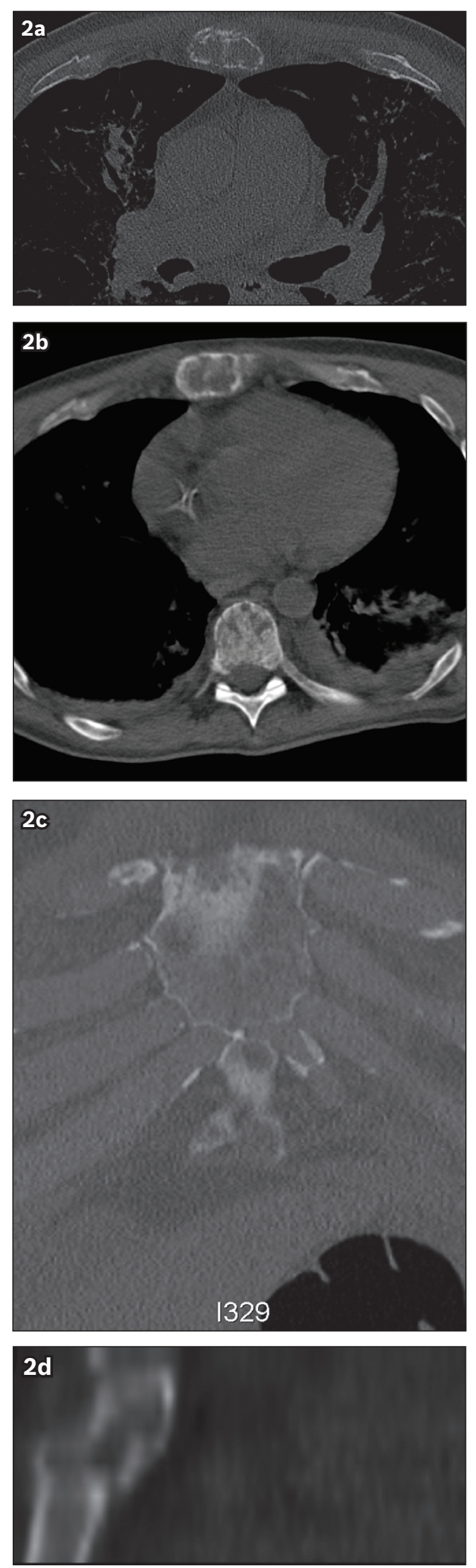

Fig. 2 Axial CT images show (a) the fracture through a lytic lesion in the sternum; and (b) the extent of solitary lytic metastasis in the sternum.

(c) Coronal CT image shows the inferior margin of the metastasis.

(d) Reconstructed sagittal CT image shows the displaced pathologic fracture of the sternum.
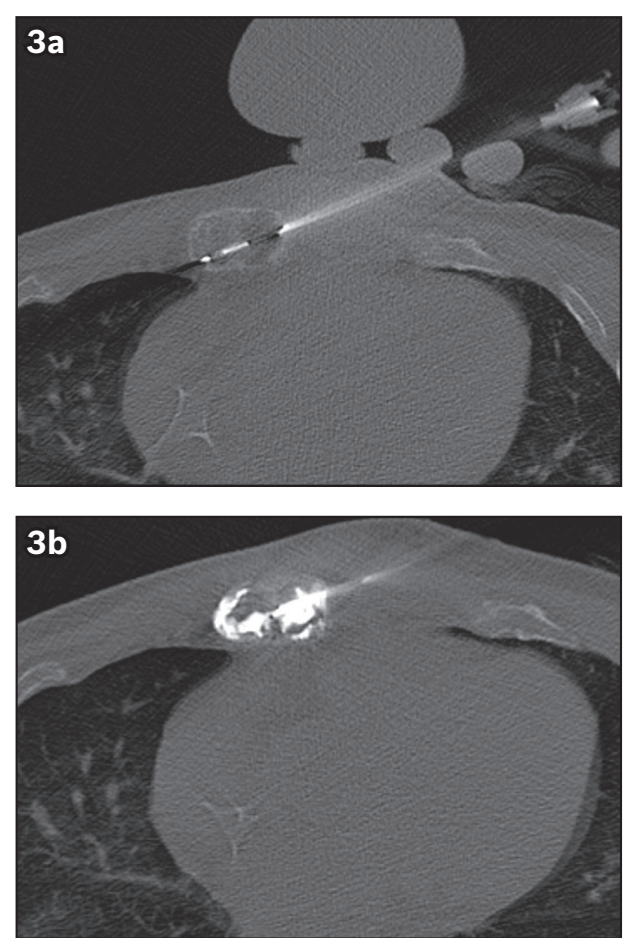

Fig. 3 Intra-procedural axial CT images show (a) the placement of the cryoprobe; a latex glove filled with warm water was placed on the skin to avoid skin freezing; and (b) the cement distribution after bone cement injection.

to act as a thermal sink to avoid skin freezing. Special care was taken in placement planning, given the proximity of the heart. The internal mammary vessels, easily seen without the use of contrast, were well out of the planned ablation zone. The chosen probe, which produces a freeze zone of $-40^{\circ} \mathrm{C}$ measuring $15 \mathrm{~mm} \times 25 \mathrm{~mm}$, completely killed cells within a zone of $-20^{\circ} \mathrm{C}$ measuring $24 \mathrm{~mm} \times 34 \mathrm{~mm}$, with less complete tissue destruction. Familiarity with the size of the ablation zone allowed accurate planning of probe placement so as to avoid the myocardium.

A 10-min freeze protocol (using a standard Argon gas system) was performed, followed by an 8 -min active thaw and a second 10-min freeze. The vertebroplasty needle was re-advanced over the cryoprobe and the probe was then withdrawn. Cementoplasty was performed using $2.8 \mathrm{~mL}$ of bone cement, with intermittent imaging performed during injection to monitor the distribution of the cement. At completion, CT examination was conducted to document the final cement distribution, after which the patient was returned to the inpatient floor with no complications (Fig. 4). The patient experienced approximately six hours of tenderness over the sternum. The next day, he reported almost $100 \%$ improvement in his pain symptoms around the anterior chest wall area, as well as resolution of postural discomfort (visual analogue scale pain score: pre-procedure 9/10; post-procedure 1/10).

\section{DISCUSSION}

External beam radiotherapy has been considered the gold standard for treatment of painful bone metastases, but it is of 


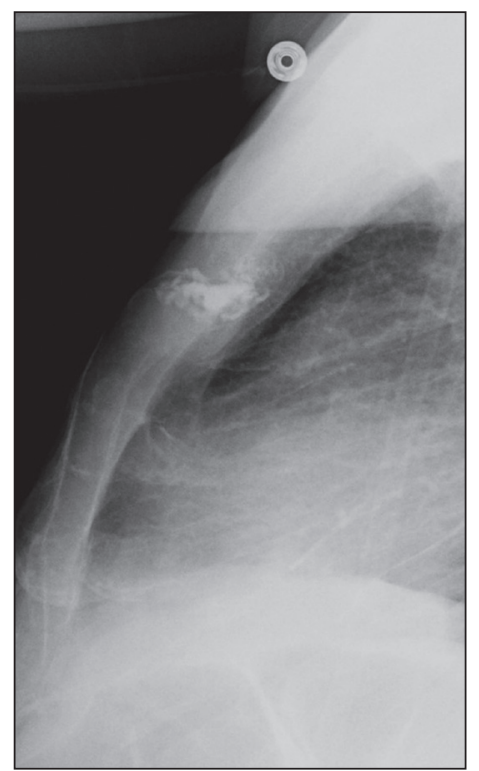

Fig. 4 Post-procedural lateral chest radiograph (coned-in view of the sternum) shows the cement in the sternum.

limited value in providing stabilisation for fracture prone or pathologically fractured areas. ${ }^{(1)}$ Radiotherapy also has the disadvantage of delayed pain relief compared to thermal ablation and cementoplasty. In fact, a significant number of patients on external beam radiotherapy do not receive any symptomatic relief at all. Bisphosphonate therapy is gaining momentum as the new standard of care for decreasing and delaying complications of osseous metastatic disease, but it too has a limited role in cases where a pathologic fracture already exists; it is also associated with a risk of renal impairment and osteonecrosis of the jaw. ${ }^{(4)}$

Pathologic fractures of the sternum have always presented a management predicament, as such fractures are difficult to adequately stabilise. Severe pain may impede the ventilatory mechanism, with symptoms that are easily exacerbated by deep breathing, coughing or even minor movement. As combined cryoablation and cementoplasty is able to stabilise the external fracture and treat the underlying lesion, it offers a potentially useful and durable palliative option in this difficult clinical scenario.

Cryoablation destroys pathologic tissue through the formation of ice crystals, the disruption of capillary flow and the induction of apoptosis. ${ }^{(5,6)}$ It is most commonly used to treat tumours of the lung, liver, breast, kidney and prostate. Recent papers have concluded that cryoablation is an effective, minimally invasive alternative to conventional therapies in the palliation of pain due to metastatic disease. ${ }^{(5,6)}$ It has also been proposed that ablative techniques be performed in combination with percutaneous cementoplasty to help provide support for and stabilisation of metastases in weight-bearing bones that are at risk of pathologic fracture. ${ }^{(7)}$

Cementoplasty alone has been used as a successful alternative for the treatment of non-fractured painful sternal lesions in the setting of multiple myeloma.(2) Combined radiofrequency thermal ablation and cementoplasty has been reported as a safe and effective treatment alternative for painful neoplastic bone lesions, ${ }^{(7)}$ and its use has been previously described in a non-fractured sternal lesion. ${ }^{(3)}$ In our patient, we provided treatment of an underlying metastatic lesion as well as stabilisation of a pathologic fracture by combining thermal ablation with cementoplasty. Ablation of tumour may also improve pain by decreasing tumour-elaborated factors that are odynogenic. ${ }^{(8)}$

Compared to RFA of soft tissue and bone lesions, cryoablation offers several notable advantages. For painful soft tissue metastases, cryoablation can be monitored visually under CT guidance as it forms a visible ice-sphere, whereas an RFA zone is more difficult to visualise. ${ }^{(9)}$ The ice-sphere can, however, be difficult to visualise with $\mathrm{CT}$ in mineralised lesions. Magnetic resonance imaging, where available, offers improved visualisation during the procedure. Cryotherapy can readily penetrate mineralised tissue, more so than RFA since calcified tissue functions as a thermal insulator impeding the conduction of heat. ${ }^{(9)}$ Cryoablation procedures are less painful than RFA, ${ }^{(6,9,10)}$ which proved to be a significant advantage in our case. The main disadvantage of cryoablation as compared to RFA is its procedural time. Due to the nature of the freeze-thaw-freeze cycle, the procedure may require the patient to be on the CT scanner for a longer duration (on average 30\% more time). ${ }^{(9)}$ The procedural time, however, varies depending on the size and location of the lesion being treated. For superficial lesions, special care should be taken to avoid thermal injury to the skin. In the present case, latex gloves filled with warm water were used as a thermal sink at the skin surface, and skin injury was thus avoided.

In summary, cryoablation combined with cementoplasty appears to offer a safe, effective and promising management option for painful pathologic fractures of the sternum.

\section{REFERENCES}

1. Agarawal JP, Swangsilpa T, van der Linden $Y$, et al. The Role of external beam radiotherapy in the management of bone metastases. Clin Oncol (R Coll Radiol) 2006; 18:747-60.

2. Zhou B, Wu CG, Gu YF, Cheng YD. Percutaneous osteoplasty for painful sternal lesion from multiple myeloma. Skeletal Radiol 2009; 38:281-5.

3. Masala S, Manenti G, Roselli M, et al. Percutaneous combined therapy for painful sternal metastases: a radiofrequency thermal ablation (RFTA) and cementoplasty protocol. Anticancer Res 2007; 27:4259-62.

4. Costa L, Major PP. Effect of bisphosphonates on pain and quality of life in patients with bone metastases. Nat Clin Pract Oncol 2009; 6:163-74.

5. Kurup AN, Callstrom MR. Ablation of skeletal metastases: current status. J Vasc Interv Radiol 2010; 21:S242-50.

6. Callstrom MR, Atwell TD, Charboneau JW, et al. Painful metastases involving bone: percutaneous image-guided cryoablation - prospective trial interim analysis. Radiology 2006; 241:572-80.

7. Munk PL, Rashid F, Heran MK, et al. Combined cementoplasty and radiofrequency ablation in the treatment of painful neoplastic lesions of bone. J Vasc Interv Radiol 2009; 20:903-11.

8. Mercadante S. Malignant bone pain: pathophysiology and treatment. Pain 1997; 69:1-18.

9. Callstrom MR, Kurup AN. Percutaneous ablation for bone and soft tissue metastases - why cryoablation? Skeletal Radiol 2009; 38:835-9.

10. Callstrom MR, Charboneau JW, Goetz MP, et al. Image-guided ablation of painful metastatic bone tumors: a new and effective approach to a difficult problem. Skeletal Radiol 2006; 35:1-15. 